\title{
Atmospheric and Oceanic Computational Science
}

First International Workshop

\author{
Adrian Sandu ${ }^{1}$, Amik St-Cyr ${ }^{2}$, and Katherine J. Evans ${ }^{3}$ \\ 1 Virginia Polytechnic Institute and State University \\ sandu@cs.vt.edu \\ 2 National Center for Atmospheric Research \\ amik@ucar.edu \\ 3 Oak Ridge National Laboratory \\ evanskj@ornl.gov
}

\section{The Workshop}

The first workshop on Atmospheric and Oceanic Computational Science brings together computational and domain scientists who develop computational tools for the study of the atmosphere and oceans. These tools are essential for understanding and prediciting weather, air and water pollution, and the evolution of the planet's climate. The dynamics of the atmosphere and of the oceans is driven by a multitude of physical processes and is characterized by a multiple spatial and temporal scales. Moreover, the computations are very large scale: present day models track the time evolution of tens of millions to tens of billions variables. These factors make atmospheric and oceanic simulations a challenging, vibrant research field with a tremendous impact on society at large.

Topics covered in this symposium include new methods for spatial and temporal discretization, parallel and high performance computing, advances with existing models, and data assimilation and observation targeting algorithms.

\section{The Papers}

Ten papers have been selected for oral presentations.

A Fully Implicit Jacobian-Free High-Order Discontinuous Galerkin Mesoscale Flow Solver by A. St-Cyr, D. Neckels proposes a discretization of the compressible Euler equations using the discontinuous Galerkin approach on collocated Gauss type grids. Time discretization uses a stiffly stable Rosenbrock W-method is combined with an approximate evaluation of the Jacobian.

Time acceleration methods for convection on the cubed sphere by R. Archibald, K. Evans, J. Drake, and J. White discusses new algorithms to overcome the scalability barriers in climate simulations. A combination of multiwavelet discontinuous Galerkin method with exact linear part time-evolution schemes can overcome the time barrier for advection equations on a sphere.

Comparison of Traditional and Novel Discretization Methods for Advection Models in Numerical Weather Prediction by C. Mavriplis, S. Crowell, D. Williams, and 
L. Wicker compares CPU time, number of degrees of freedom and overall behavior of solutions for finite difference, spectral difference and discontinuous Galerkin methods on several model advection problems relevant to numerical weather prediction.

A non-oscillatory advection operator for the compatible spectral element method by M.A. Taylor, A. St-Cyr, and A. Fournier presents the development of a monotone or sign-preserving advection operator based on a spectral element formulation, and implemented via the highly scalable cubed-sphere atmospheric dynamical core package HOMME into the Community Climate System Model (CCSM).

Simulating Particulate Organic Advection Along Bottom Slopes to Improve Simulation of Estuarine Hypoxia and Anoxia by P. Wang and L.C. Linker presents an approach to move volatile solids from the shoals to the channel by simulating movement of particulate organics due to slopes based on an example in the Chesapeake Bay eutrophication model. Implementations for the simulation of this behavior in computer parallel processing are discussed.

Explicit time stepping methods with high stage order and monotonicity properties by E.M. Constantinescu and A. Sandu introduces a three and a four order explicit time stepping methods with high stage order and favorable monotonicity properties. The proposed methods are based on general linear methods, and are generalizations of both Runge-Kutta and linear multistep methods.

Improving GEOS-Chem Model Tropospheric Ozone through Assimilation of Pseudo Tropospheric Emission Spectrometer Profile Retrievals by K. Singh, P. Eller, A. Sandu, K. Bowman, D. Jones, and M. Lee discusses a recently-developed adjoint model of GEOS-Chem global chemical transport model, and 4D-variational data assimilation studies used to improve of 2006 summer time distribution of global tropospheric ozone through assimilation of pseudo profile retrievals from the Tropospheric Emission Spectrometer (TES).

Chemical Data Assimilation with CMAQ: Continuous vs. Discrete Advection Adjoints by T.Y. Gou, K. Singh, and A. Sandu discusses a new implementation of the adjoint of Community Multiscale Air Quality (CMAQ) modeling system. The construction of discrete adjoint code is derived from the forward model code with the aid of automatic differentiation.

A Second Order Adjoint Method to Targeted Observations by H.C. Godinez and D.N. Daescu studies the role of the second order adjoints in observation targeting strategies. The dominant eigenvectors of the Hessian matrix indicate the directions of maximal error growth for a given targeting functional. These vectors are a natural choice to be included in the targeting strategies given their mathematical properties.

A scalable and adaptable solution framework within components of the Community Climate System Model by K.J. Evans, D.W.I. Rouson, M.A. Taylor, A.G. Salinger, W. Weijer, and J.B. White III implements a a framework for a fully implicit solution method into the High Order Methods Modeling Environment (HOMME), and the Parallel Ocean Program (POP) model of the global ocean. Both of these models are components of the Community Climate System Model (CCSM). 